\title{
An Insight into Scheduling Algorithms for Mobile Grid: A Survey
}

\author{
T. Vigneswari*, M. A. Maluk Mohamed \\ M.A.M College of Engineering, Tiruchirappalli, India. \\ * Corresponding author. Tel.: 9790222623; email:jayasuriyaus@yahoo.com \\ Manuscript submitted February 1, 2016; accepted May 7, 2016. \\ doi: $10.17706 /$ jcp.12.5.433-441
}

\begin{abstract}
The integration of mobile devices with grid computing pioneered a new paradigm termed as Mobile grid computing. This concept has engrossed many researchers to address the issues in utilizing the mobile resources in grid computing. Resource management and scheduling is one of the major concerns in mobile grids due to the impending nature and dynamic scale of resources. Scheduling in grid computing is NP complete and many scheduling strategies have been introduced for the efficient utilization of resources. But these algorithms cannot be applied to mobile grids. Many strategies have been developed for the optimal scheduling of resources in mobile grid. In this paper we provide an insight into the scheduling algorithms that have been tailor made for mobile grids.
\end{abstract}

Key words: Mobile Grid, scheduling, Grid computing, resource management.

\section{Introduction}

Ref. [1], [2] There is a hunger for enormous amount of resources among applications like scientific research, weather forecasting or a simulation for new aircraft design carried out over all around the world .The term resource mentioned here is not only mere computing power but also refers to storage, network bandwidth ,file systems and instruments like telescope. The common distributed concepts cannot coordinate heterogeneous resources from different sites. The sharing was limited due to centralized control. Grid was seen as the big distributed computing paradigm for complex applications said above. Grid pools resources that are pervasive dependable, consistent and inexpensive. Sharing of resources dynamically in a coordinated fashion is possible by means of Virtual organization Resources in a grid belongs to multiple organizations, follow different security policy, available on demand, and have fluctuations in availability. Other distributed applications such as clusters, web, Peer to peer systems, Application \& storage providers also look like Grid. A Check list for grid computing was given by foster [3] which states that , resources should not have centralized control, open standards should be followed and QoS should be provided. The above given list differentiate grid vividly from others. Even though a thorough reading on grid computing poses an image that grid can do anything, still there are barriers to overcome. Due to many impending reasons [4] grid is used only among a small group of peoples. Making a grid to work properly is a big task due to lack in basic functionality. Heterogeneity among the resources in a grid raises unpredictable problems which affect many decisions from fault management to resource selection including security problems sociopolitical and economical reasons also add oil to the burning lamp. There is a strong need for standard software tools to adapt to grid environment with ease of deployment .As sharing is the major key word used in grid ,benefits to the resource providers should be unambiguous with the aid of proper 
accounting system.

The first generation grids [5] allowed users to simply share resources. The second generation of grids focused on middleware to align other technologies with the grid and in the third generation converging the web with the grid is made possible. Now the recent trends in grid computing incline towards pervasiveness and self management and they are classified as emerging grids based on four desired characteristics such as accessibility, interactivity, user centric and manageability. The four main groups of grid under this classification are accessible grids, user centric interactive and manageable grids. Mobile grids come under the accessibility grids by differentiating itself from traditional grids with the support for mobility of clients, services or both.

Grid is evolving as a SOA for securely orchestrating and sharing stateful web services and resource across distributed organizations. Mobile grids make grid service available and accessible anytime, anywhere from mobile devices. Limitations and constraints of mobile devices [6] are Low processing power, Low built in memory, Less storage capacity compared to desktops, Less battery life, Size restriction in display size, Poor quality display, Unstable network due to intermittent connectivity and poor bandwidth. This limitations can be compensated when combined with grid .

Mobile devices and grid when integrated in a mobile grid environment complements each other. To the grid, Mobile devices increase the accessibility of grid as more number of mobile devices are available. Three main approaches for mobile grid client are java based, net based and proxy based. Despite the fact that mobile devices have less computing power and low storage space, the number of mobile devices is huge in number .Almost every individual in the globe owns a collection of devices like smart phones, laptop, PDA etc. These devices can provide the service needed by the grid user when aggregated. Mobile devices can offload the resource demanding work to powerful devices in grid. The mobile devices can collaborate with themselves or with another static device in the grid .It improves the user experience, convenience and contextual relevance [7], [8]. Thus mobile grid is emerging as a promising strategy for the better utilization of scattered resources. The constraints related to mobility, availability of mobile resources connectivity, security breaches energy sources etc hinder the wide spread usage of mobile grid.

\section{Scenario of Resource Management and Scheduling in Mobile Grid}

Three different models for resource management has been discussed in [9]. The Hierarchical Model which confines to architecture followed in most existing systems. The Abstract Owner Mode follows an order and delivery model for resource sharing. But it ignores the infrastructure to focus on long-term goals. The Computational Market/Economy Model follows economic model in resource discovery and scheduling that can co-exist or work with current systems and incarcerate the essence of both hierarchical and abstract owner models.

Job scheduling and resource management is one of the major issues faced by mobile grid system. The conventional job scheduling policies of a normal grid cannot be adapted as it is in the mobile gird environment where the mobility of devices and constraints faced due to the mobile devices should be given due consideration. In this paper we provide a detailed survey about various scheduling algorithms that are available in the literature which solves the scheduling problem from various perspectives.

Resource management system [10], [11] maintains a list of resources available for the users. The resources are varied in nature like processors, Network bandwidth, and disk storage, application software's etc. The users may request for resources directly or indirectly to the grid. Generally this is termed as task submission or job submission. Upon receiving the request the resource management system schedules the resources to job. Resource management in grid becomes difficult due to heterogenic nature of resources, ownership from multiple domains and irregularities in communication. The major challenges faced are 
scalability and adaptability. This is in the case of a normal grid [12]. Apart from this, scheduling in mobile grid is affected by the constraints of mobile devices also like mobility and availability of resources.

Job scheduling in a mobile grid environment becomes even more convoluted due to the constraints of mobile resources that are involved. The overall system scalability and current availability of individual resources should be considered during scheduling. Along with these factors cost, performance and reliability of resources also should be taken into account. In an unpredictable environment like mobile grid, job monitoring and execution are very difficult. The completion of the jobs [13] is also assured by rescheduling jobs and job migration as well as job replication and co-scheduling. As in conventional grids, scheduling is NP complete [14], [15]. Due to this verity the scheduling algorithm has match the job request with the resources optimally such that resource utilization is minimum and output is maximum.

\section{Survey of Scheduling Algorithms}

In this section we have given a brief account of scheduling algorithms involved in mobile grid environment. We have also categorized the algorithms based on the scheduling criteria such as availability, energy consumption and battery power of resource, mobility, fault tolerance etc.

\subsection{Energy Consumption and Battery Power}

Energy efficiency of the resources is an important factor as the successful execution of the running in a mobile node is determined by the available energy. Optimal energy efficient resource management should be done to maximize the utilization of mobile grid. Majority of the mobile resources relies on battery power and hence algorithms considering the battery power have emerged in the mobile grid scenario.

A scheduling algorithm based on the mobility and battery power within a cell is proposed in [16]. The battery power is calculated based on C-rate of the resource. The mobility is based on movement information such as type, pattern and direction within the zone. The requirements are checked dynamically as the requirements vary over time. A resource prediction technique is incorporated for this purpose which predicts the information about resource based on battery power and mobility. The same author has proposed an extension of the above said algorithm [17] based on availability of battery power and mobility of resources within a zone. The same prediction mechanism has been adapted within a zone for scheduling the resources. The performance of the system varies in direct proportionate with the residence time of the resource with in a zone.

A two level Hierarchical scheduling is proposed in [18]. A revised min-min is algorithm is used in the second level so as to decrease the energy consumption during communication and computation. Finally, a scheduling decision is arrived based on task deadline. The results show that overall energy utilization is improved and there was also a significant decrease in failure ratio of tasks

Scheduling of jobs based on Genetic Algorithm in mobile grid is proposed in [19] to provide better scheduling than conventional methods. The criterion such as inferior reliability, limited capacity and capability of mobile devices are taken into account for scheduling the jobs. Further to enhance reliability of the job execution, the replication scheme $t$ is used. The results show that the job completion time is decreased and resource utilization is improved by utilizing this method.

An energy aware scheduler is proposed in [20]. This scheduler works in two phases. The first phase calculates the resource performance and also the remaining battery time. Based on this the devices are ranked then they are scheduled to the task using FCFS algorithm.

The utility of a grid application doesn't depend only on computing and communicating capacity of a resource but also includes energy consumption by the resource. A price based modeling [21] is derived for energy constrained resources based on factors such as resource capacity, application budget and completion times. This algorithm derives two utility functions for maximizing the benefits of application 
and optimizing the venue of the resources to produce an optimized scheduling. This scheduling strategy reduces the energy consumption of resources and also improves the utility of the application. This algorithm also ensures the battery life time and deadlines of the application.

The limited capacity and inferior reliability of the resource pose a problem during scheduling of jobs. Job replication improves the reliability of executing a job in the grid but at the same time execution of useless replicas should be avoided. A scheduling method based on genetic algorithm was proposed in [22] to schedule the replicated jobs. This algorithm effectively minimizes the completion time and overhead caused by the job replication.

The most resourceful resources are selected for scheduling by using Delayed reply and $\mathrm{SbV}$ algorithms .Then a protocol called resource DICHOTOMY [23] schedules the resources by mitigating the overhead of discovery messages exchanged among the nodes. The protocol works in a peer-to-peer fashion, irrespective of the routing protocols used. This protocol also provides implicit load balancing.

\subsection{Mobility}

Mobility in a mobile environment may be Session mobility, Terminal mobility, User mobility and Service mobility. User mobility [24] involves the movement of the user and the identity or the property of the resource is not changed. Session mobility [25] allows the movement of communicating end-points to support accidental long network access outages and predefined disconnection times.

In case of terminal mobility [26], the resource used by the user changes from time to time thus the underlying middleware has to be aware of the changes. The location of the services offered is changed in Service Mobility [27] but all the essential communication between the two parties are saved by communication end point. This is done to provide seamless availability of service. A game theoretic pricing strategy has been proposed for mobile grids in [28] for better resource scheduling. This bargaining game determines a fair pricing strategy which allocates the job efficiently to the mobile devices. Maximization of revenue to the user is the major objective of this algorithm.

A mobility model for scheduling in mobile grid was proposed in [29]. This paradigm predicts the time duration for which a resource will reside in a particular domain. This duration was calculated based on the past behavior of the resource. Based on this, resource which doesn't move often are scheduled and improves the performance of the scheduler.

A scheduling algorithm which considers both mobility and load balancing is suggested in [30]. The resource availability is classified into three types. Further they are classified into nine groups. A Multilevel queue with priority is proposed for job scheduling. Jobs are prioritized based on their communication interval and assigned to the resources according to their availability.

A scheduling mechanism considering the stability of mobile resources was proposed in [31]. The major objective of this algorithm is $t$ decrease the overhead caused by job replication thus minimizing the completion time Genetic algorithm is employed for this purpose and results outperform the conventional scheduling in achieving the above said objective.

The latency incurred during handover was considered in the scheduling algorithm proposed in [32] \& [33]. This algorithm doesn't take into account, the wastage of network, computational and energy of resources in a mobile node during the abortion of a task.

A scheduling paradigm based on mobility of nodes was presented in [34]. A centralized scheme tracks the location of nodes and estimates the availability of mobile nodes. Based on this information, mobile nodes are scheduled. However, fault tolerance is not dealt in this algorithm.

The submitted jobs are differentiated into computing focused and communication focused jobs in [35]. This paper proposes a mobility aware load balanced algorithm in which scheduling is done based on above said job types. The computing focused jobs are submitted to resources with shorter round trip, high CPU 
speed and capacity. The communication focused jobs are submitted to resources with low mobility and high reliability. This algorithm improves the network performance also. The downtime may be planned or unplanned. The resource selection and allocation depends highly on the availability.

A two phase scheduling algorithm which groups the resources based on the availability [36] in the first phase and in the second phase scheduling is done by using genetic algorithm based on parameters such as mobility, availability and job completion. This algorithm minimizes the job completion time.

A scheme proposed in [37] uses multi agents for providing better scheduling solution. It proposes two agencies Grid Information Service (GIS) and resource broker (RB). Each agency has its own agent to carry out their task. GIS agent provides information about the grid infrastructure. The RB agent recommends appropriate resource for the task based on the information received from GIS. If a device moves out of the MSC during execution, it is dynamically rescheduled by the RB. The job completion time and execution rate are improved by using this algorithm.

\subsection{Intermittent Connections}

Intermittent connections is one of the constraint faced when using mobile resources.. Wireless link failure, battery power exhaustion, and abnormal shutdown or out of coverage may lead to the connection between mobile device and grid interrupted. The job running in a mobile grid will be adversely affected by the intermittent connection so due consideration should be given [38].

An algorithm adapting to the intermittent connection of resources was proposed in [39]. This MGRR algorithm considers all the independent jobs with same length.The major objective of this algorithm is to minimize the total processor cycle consumption. This algorithm does not use any prediction information. It uses rescheduling and replication for achieving the desired performance.

The stability of network (intermittent connection) connection with the mobile terminal plays an inevitable role in task scheduling of mobile grids. With an objective of reducing the total execution time and task completion time in above said case, Qing Jiang et al [40] have proposed scheduling algorithm based on genetic algorithm. Simulation examples vividly show that the algorithm also improves the resource utilization ratio and improve grid task analysis efficiency. The overall lifespan changes drastically when the number of tasks increases over time.

The performance of mobile grid was affected by intermittent connections of resources due to user mobility. Adapting synchronous message delivery becomes tedious due to this characteristic of mobile grid. To overcome this problem mobile agent based architecture to support asynchronous message delivery and location management was proposed in [41]. In this method, the resources are classified into full availability, partial availability, and unavailability so that users' mobility can be taken into account. An $\mathrm{n}$ adaptive load-balancing technique based on the above said classification was propose which uses adaptability based on the multi-level feedback queue to handle the job type change. The results of this work shows significant improvement of execution time when compared with algorithms not taking into account of considering mobility and adaptive load-balancing.

\subsection{Fault Tolerance}

Fault tolerance as a metric that is adjusted in the Grid infrastructure based on the reliability model of its resources.

An adaptive replication was used for avoiding faults in an active manner. This algorithm shows performance improvement in terms of execution times compared with the algorithm that doesn't compares grouping and fault tolerance [42]. A group-based fault tolerance scheduling algorithm [43] which categorize the devices into several groups by considering characteristics parameters of mobile grid.

A group based fault tolerance algorithm introduced in [44] improves the resource utilization and task 
reliability. This was achieved by scheduling by grouping the resource and also applying fault tolerance algorithm. The outcome of this algorithm was compared with group-based scheduling algorithm without fault tolerance and the group-based scheduling algorithm with check pointing.

Priority based scheduling was proposed in [45]. The prioritization was based on QOS attributes. This scheme improves the reliability of the system and fault tolerance.

The Scheduling algorithm introduced in [46] allows decision making done locally by the worker peers. The new jobs are accepted by peers only if it is capable of processing it and it is not overloaded. Load balancing and fault tolerance are taken care

Many other works were seen in the literature which aims at improving the scheduling by applying various strategies.

The performance of basic scheduling methodologies like Abort-Reschedule, Installments, Groups policy is discussed in [47]. They are evaluated based on metrics like Response Time, resource waste, speedup and task completion time

A scheduling algorithm to decrease the total response time was proposed in [48]. In this algorithm, the mobile nodes are sorted in the order of increasing expected response time. The scheduler selects the mobile nodes that yield minimum total response.

A scheduling algorithm in [49] aims at achieving multiple objectives such as job completion, lateness minimization. Genetic algorithm with Niching-pareto implementation was utilized for efficient scheduling. The niche-pareto reduce the effect of genetic drift found in conventional GA and allows multiple objectives to be solved parallel.

This paper [50] experimented the possibility of including the mobile devices in grid computing for scheduling the task. The FCFS policy is used. The job which is preempted can rejoin the queue and again it is scheduled. Heuristic scheduling algorithms such as FCFS, OLB, MCT, Min-Min and Online were used for scheduling the tasks to mobile devices. The results have shown that utility is better for mobile devices.

The additional parameters that should be taken into account during grid scheduling have been listed in [51]: start and end time of the nodes' availability (TS, TE), effective (available) time of the node (TEFF), processor speed (CPU), number of instructions ( $\mathrm{N}$ ins), task size (L), network throughput (rate) (R), number of hops (N), round trip time (RTT) and the battery energy available (B). Five scheduling algorithms have been proposed by the authors. Greedy algorithm and simulated annealing algorithms were modified to suit the scheduling in mobile adhoc grids. They have also proposed three new algorithms namely scheduling based on hop-count, scheduling based on RTT, and Scheduling based on overall tasklets transmission and execution time.

Resource reliability is considered as criteria for scheduling in [52]. The reliability is evaluated from task completion time and energy consumption. The reliability was also calculated in different manner for urgent and non urgent tasks. The min -min algorithm is applied to this which uses the reliability factor to schedule the resources .The algorithm minimizes energy consumption, decrease task completion time and provides load balancing.

\section{Conclusion}

Optimal Scheduling of resources improves the overall performance of the grid environment. This fact applies for mobile grid environment also. In this work, a brief account of scheduling algorithms currently available in literature that are utilized in a mobile grid environment is given. The constraints of mobile devices like availability, mobility, intermittent connectivity, fault tolerance, etc has been considered as criteria for selecting resources during scheduling. Many conventional algorithms have been enhanced to suit the dynamic mobile environment from this study we conclude that there is a room for the application of 
metaheuristics algorithm which involves swarm intelligence in volatile environment like mobile grids would further improve the performance of scheduling.

\section{References}

[1] Foster, I., Carl, K., \& Steven, T. (2001). The anatomy of the grid: Enabling scalable virtual organizations. International Journal of High Performance Computing Applications, 15(3), 200-222.

[2] Foster, I. \& Kesselman, C. (1999). The Grid: Blueprint for a New Computing Infrastructure. Morgan Kaufmann.

[3] Foster, I. (July 22, 2002). What is the Grid? Daily News and Information for the Global Grid Community, 1(6).

[4] Schopf, J., \& Nitzberg, B. (Aug. 2002). Grids: Top ten questions. Programming Special Issue on Grid Computing, 10(2), 103-111.

[5] Kurdi, H., Maozhen, L., \& Hamed, A.-R. (2008). A classification of emerging and traditional grid systems. Distributed Systems Online, 9(3).

[6] Phan, T., Lloyd, H., \& Chris, D. (2002). Challenge: Integrating mobile wireless devices into the computational grid. Proceedings of the 8th Annual International Conference on Mobile Computing and Networking.

[7] Parmar, K. B., Jani, N. N., Shrivastav, P. S., \& Patel, M. H. Mobile grid computing: Facts or fantasy? Int. Journal of Multidisciplinary Science and Engineering, 4(1).

[8] Bichhawat, A., \& Joshi, R. C. (2010). A survey on issues in mobile grid computing. Int. J. of Recent Trends in Engineering and Technology, 4(2).

[9] Buyya, R., Steve, C., \& David, D. (2000). Architectural models for resource management in the grid. Grid Computing-GRID 2000, 18-35. Springer Berlin Heidelberg.

[10] Krauter, K., Rajkumar, B., \& Muthucumaru, M. (2002). A taxonomy and survey of grid resource management systems for distributed computing. Software-Practice and Experience, 32(2). 135-164.

[11] Nabrzyski, J., Jennifer, M. S., \& Weglarz, J. (2012). Grid resource management: State of the art and future trends. 64, Springer Science \& Business Media.

[12] Cao, Junwei, et al. (2002). ARMS: An agent-based resource management system for grid computing. Scientific Programming, 10(2), 135-148.

[13] Litke, A., Skoutas D., \& Varvarigou, T. (2004). Mobile grid computing: Changes and challenges of resource management in a mobile grid environment. Proceedings of Practical Aspects of Knowledge Management.

[14] Moreno, R. (2003). Job scheduling and resource management techniques in dynamic grid environments. Proceedings of 1st European Across Grids Conference.

[15] Abraham, A., Buyya, R., \& Nath, B. (2000). Nature's heuristics for scheduling jobs on computational grids. Proceedings of International Conference on Advanced Computing and Communications.

[16] Stephen, V. S., \& Mary, S. B. S., (2011). Mobility and battery power prediction based job scheduling in mobile grid environment. Proceedings of the International Conference on Parallel, Distributed Computing technologies and Applications (p. 312).

[17] Stephen, V. S., \& Mary S. B. S. (2012). Zone Based job scheduling in mobile grid environment. International Journal of Grid Computing \& Applications, 3(2).

[18] Huang, C.-Q., Zhu, Z.-T., Wu, Y.-H., \& Xiao, Z.-H. (2006) Power-Aware hierarchical scheduling with respect to resource intermittence in wireless Grids. Proceedings of the Fifth International Conference on Machine Learning and Cybernetics (pp. 693-698). Dalian.

[19] Chin, S., Taeweon, S., et al. (2012). A low-overhead scheduling scheme based on genetic algorithm in 
mobile grids. Journal of Internet Technology, 13(1), 137-146

[20] Juan, M. R., Alejandro, Z., \& Marcelo, C. (2010). Mobile grid seas: Simple energy-aware scheduler. Proceedings of 3rd High-Performance Computing Symposium.

[21] Li, C. L., \& Li, L. Y. (2010). Energy constrained resource allocation optimization for mobile grids. Elsevier, Journal of Parallel Distrib. Comput., 70, 245-258.

[22] Chin, S., Taeweon, S., \& Yu, H. C. (2009). Genetic algorithm based scheduling method for efficiency and reliability in mobile grid. Proceedings of the 4th International Conference on Ubiquitous Information Technologies \& Applications.

[23] Gomes, A. T. A., Ziviani, A., Lima, L. S., \& Endler, M. (2007). Dichotomy: A resource discovery and scheduling protocol for multihop ad hoc mobile grids. Proceedings of 7th IEEE Int. Symp. on Cluster Computing and the Grid (pp. 719-724).

[24] Landfeldt, B., et al. (1999). SLM, a framework for session layer mobility management. Proceedings of Eight International Conference on Computer Communications and Networks.

[25] Salkintzis, A. K., Chad, F., \& Rajesh, P. (2002). WLAN-GPRS integration for next-generation mobile data networks. Wireless Communications, 9(5), 112-124.

[26] Migliardi, M., \& Vaidy, S. (2001). Plug-ins, layered services and behavioral objects: Application programming styles in the HARNESS metacomputing system. Future Generation Computer Systems, 17(6), 795-811.

[27] Migliardi, M., et al. (1998). Dynamic reconfiguration and virtual machine management in the Harness metacomputing system. Computing in Object-Oriented Parallel Environments, 127-134. Springer Berlin Heidelberg.

[28] Preetam, G., Nirmalya, R., Das, S., \& Basu, K. (2005). A pricing strategy for job allocation in mobilegrids using a non-cooperative bargaining theory framework. Special Issue on Design and Performance of Networks for Super-Cluster and Grid-Computing, 1366-1383.

[29] Farooq, U. \& Khalil, W. (2006). A generic mobility model for resource prediction in mobile grids. Proceedings of the International Symposium on Collaborative Technologies and Systems.

[30] Lee, J., Lee, H., Chung, K., \& Yu, H. (2009). Balanced scheduling algorithm considering availability in mobile grid. 212-222, Springer, Heidelberg.

[31] Yu, H. C., et al. (2009). Genetic algorithm based scheduling method for efficiency and reliability in mobile grid. Proceedings of the 4th International Conference on Ubiquitous Information Technologies \& Applications.

[32] Kurkovsky, S., et al. (2003). Wireless grid enables ubiquitous computing. ISCA PDCS, 399-404.

[33] Kurkovsky, S., Bhagyavati, et al. (2004). A collaborative problem-solving framework for mobile devices. Proceedings of the 42nd Annual Southeast Regional Conference (pp. 5-10). ACM Press.

[34] Ghosh, P., Roy, N., \& Das, S. K. (2007). Mobility-aware efficient job scheduling in mobile grids. Proceedings of the 7th IEEE Symposium on Cluster Computing and the Grid (pp. 701-706). IEEE Computer Society.

[35] Vimala, S., \& Sasikala, T. (2014). Mobility Aware load balanced scheduling algorithm for mobile grid environment. International Review on Computers and Software, 9(4), 687-693.

[36] Saravanan, G., \& Gopalakrishanan, V. (2014). Improved genetic algorithm for group-based job scheduling in mobile grids. Journal of Theoretical \& Applied Information Technology, 67(2).

[37] Birjea, M. N., \& Manvi, S. S. (2012). Agent based economic scheme for seamless job scheduling in bandwidth constrained wireless grids. International Journal of Grid and Distributed Computing.

[38] Ju, H. J., Du, L. J., \& Cui, W. P. (2010). Middleware framework of mobile grid. Proceedings of Annual Conference of China Institute of Communications. 
[39] Du, L. J., \& Yu, Z. W. (2010). Scheduling algorithm with respect to resource intermittence in mobile grid. Proceedings of 2010 6th International Conference on Wireless Communications Networking and Mobile Computing.

[40] Qing, J., Wu, X. L., \& Yang, H. L. (2012). Task scheduling based on genetic algorithm in mobile grid. Proceedings of International Conference on Computer Science and Service System.

[41] Lee, J., et al. (2014). Mobility-aware balanced scheduling algorithm in mobile grid based on mobile agent. The Knowledge Engineering Review, 29(4), 409-432.

[42] Litke, A., et al. (2007). Efficient task replication and management for adaptive fault tolerance in mobile grid environments. Future Generation Computer Systems, 23(2), 163-178.

[43] Lee, J. H., et al. (2010). Group-based scheduling algorithm for fault tolerance in mobile grid. Security-Enriched Urban Computing and Smart Grid, 394-403, Springer Berlin Heidelberg.

[44] Lee, J. H., et al. (2012). A mobile device group based fault tolerance scheduling algorithm in mobile grid. Embedded and Multimedia Computing Technology and Service, 485-492, Springer Netherlands.

[45] Litke, A., et al. (2009). Fault tolerant and prioritized scheduling in OGSA-based mobile grids. Concurrency and Computation: Practice and Experience, 21(4), 533-556.

[46] Hummel, K. A., \& Jelleschitz, G. (2007). A robust decentralized job scheduling approach for mobile peers in ad-hoc grids. Proceedings of the 7th IEEE Symposium on Cluster Computing and the Grid (pp. 461-470). IEEE Computer Society.

[47] Katsaros, K., \& George, C. P. (2008). Evaluation of scheduling policies in a mobile grid architecture. International Symposium on Performance Evaluation of Computer and Telecommunication Systems.

[48] Park, S.-M., Ko, Y.-B., \& Kim, J.-H. (2003). Disconnected operation service in mobile grid computing. ICSOC, 499-513.

[49] Benedict, S., \& Vasudevan, V. (2008). A niched pareto ga approach for scheduling scientific workflows in wireless grids. Journal of Computing and Information Technology, 16(2), 101-108.

[50] Ashish, C., Bibhudatta, S., \& Ashok, K. T. (2011). Efficient Task Scheduling using Mobile Grid.

[51] Cilku, B., \& Aksenti, G. (2009). New algorithms for efficient scheduling in grid Ad-Hoc networks. Proceedings of the ITI 2009 31st International Conference on Information Technology Interfaces (pp. 22-25).

[52] Liu, L., \& Li, C. L. (2009). Mobile grid task scheduling considering resource reliability. Computer Network and Multimedia Technology.

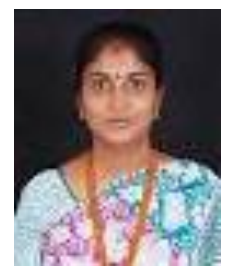

T. Vigneswari is working as an Assistant Professor in Kings College of Engineering Punalkulam, Tamilnadu, India. She is pursuing her Ph.D in Anna University. She has completed her B.E and M.E in computer science and engineering. She is a member in professional societies like ISTE, Indian Science Congress etc.

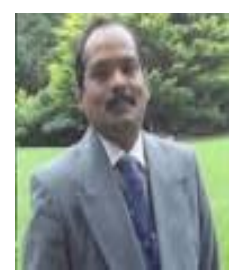

M. A. Maluk Mohamed is Professor and Research Guide of CSE Department, M.A.M College of Engineering. He has completed his Ph.D. in Indian Institute of Technology Chennai, India, master of engineering in Regional Engineering College, Tiruchirapalli, India, bachelor of engineering from Mookambigai College of Engineering College, Bharathidasan University, Tiruchirapalli, India. So far six scholars have obtained doctoral degree under his guidance. 